\title{
Volumen testicular en pacientes adultos operados de criptorquidia en edad infantil y su efecto sobre la paternidad
}

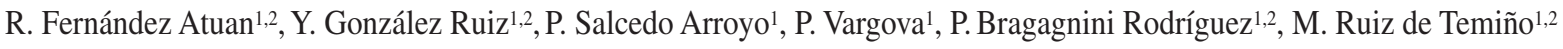

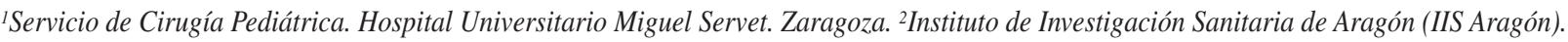

\section{ReSUMEN}

Objetivos. Determinar el efecto de la orquidopexia sobre el volumen testicular. Determinar si la edad de intervención afecta el volumen testicular. Determinar si la paternidad se asocia al volumen testicular.

Métodos. Pacientes nacidos entre los años 1961 y 1985, intervenidos de criptorquidia por el Servicio de Cirugía Pediátrica del Hospital Universitario Miguel Servet, tomando datos de la localización del teste y aspecto macroscópico. Se realizan ecografías testiculares de control y encuestas de paternidad. Realizamos un estudio inicial descriptivo y posteriormente inferencial.

Resultados. La ecografía se realizó con una media de 14,9 años postoperatorios en 216 unidades testiculares y la encuesta de paternidad con una media de 41,9 años postoperatorios a 157 participantes. Existen diferencias significativas $(\mathrm{p}=0,0038)$ en la distribución del volumen testicular según disyunción del epidídimo. Hay correlación lineal entre mayor edad de tratamiento quirúrgico y menor volumen testicular, sin llegar a significancia estadística. Se observan diferencias significativas $(p=<0,0001)$ en el volumen testicular según grupos de operados y no operados, como también entre los unilaterales y los bilaterales. No se observan diferencias en índices de paternidad según volumen testicular.

Conclusión. El teste intervenido presenta un volumen testicular menor que el teste de descenso normal. Una mayor edad de tratamiento quirúrgico puede contribuir a un menor volumen final del teste. Los testículos con disyunción epidídimo testicular completa tienen menor volumen total. No observamos relación entre el volumen testicular e índices de paternidad. Más estudios a largo plazo son necesarios.

Palabras Clave: Testículo no descendido; Criptorquidia; Volumen testicular; Orquidopexia.

DOI: $10.54847 /$ cp.2022.01.05

Correspondencia: Dr. Rafael Fernández Atuan.

E-mail: raferat@gmail.com

Trabajo presentado como comunicación en el LVIII Congreso de Cirugía

Pediátrica de la SECIPE. Vigo 2019.

Presentado como póster en el Vigésimo Congreso Europeo de Cirugía

Pediátrica (EUPSA). Belgrado 2019.

Recibido: Octubre 2021

Aceptado: Noviembre 2021

\author{
TESTICULAR VOLUME IN ADULT PATIENTS UNDERGOING \\ CRYPTORCHIDISM SURGERY IN CHILDHOOD, AND IMPACT \\ ON PATERNITY
}

\begin{abstract}
Objective. To determine the impact of orchiopexy on testicular volume. To determine whether age at surgery impacts testicular volume. To determine whether paternity is associated with testicular volume.

Methods. Patients born between 1961 and 1985 who had undergone cryptorchidism surgery at the Pediatric Surgery Department of Miguel Servet University Hospital were included. Testis location and macroscopic appearance data were collected. Control testicular ultrasonographies and paternity surveys were carried out. Initially, the study was descriptive, and subsequently, inferential.

Results. Ultrasonography was performed in 216 testicular units a mean of 14.9 years following surgery, whereas the paternity survey was conducted among 157 respondents a mean of 41.9 years following surgery. There were significant differences $(p=0.0038)$ in testicular volume distribution according to epididymal dissociation. There was a linear correlation between older age at surgery and lower testicular volume, but without statistical significance. Significant differences $(p=<0.0001)$ in testicular volume according to groups -operated and non-operated-, as well as between unilateral and bilateral cases, were found. No differences in paternity rates according to testicular volume were noted.

Conclusion. Operated testes have lower volumes than normally descended testes. Older age at surgery may contribute to lower final volumes. Testes with full epididymal-testicular dissociation have lower total volumes. No relation between testicular volume and paternity rates was found. Further long-term studies are required.
\end{abstract}

KeY Words: Undescended testis; Cryptorchidism; Testicular volume; Orchiopexy.

\section{INTRODUCCIÓN}

La criptorquidia según Hutson en 2010(1) es la ausencia del testículo en la bolsa escrotal y que, en el caso de ser palpable, no puede ser manipulado hasta el fondo del saco escrotal sin aplicar tensión en el cordón espermático, habitualmente regresando de inmediato a su posición anormal al liberar la tensión. 
La criptorquidia es la anomalía congénita más frecuente en los niños. En recién nacidos a término, la incidencia es de $3-5 \%{ }^{(1-3)}$. A medida crecen en el primer año, en el $70 \%$ de los casos, el teste desciende a bolsa espontáneamente. $\mathrm{Al}$ año, la prevalencia oscila alrededor del $1 \%$ y esta cifra persiste en la adultez ${ }^{(3,4)}$. Schneuer encontró una incidencia de testes intervenidos de 9,6/1.000 nacidos vivos ${ }^{(5)}$.

Un teste fuera de su localización normal está sujeto a alteraciones histológicas. Según estudios realizados por Hadziselimovic, hay una reducción en el número de gonocitos a espermatogonias AD (tipo A oscuras) y, por tanto, una alteración de la espermatogénesis ${ }^{(6)}$. Verkauskas, en estudio prospectivo, reporta que del $9-15 \%$ de pacientes unilaterales y hasta el $46 \%$ de pacientes bilaterales desarrollan oligospermia o azoospermia ${ }^{(7)}$. Otras alteraciones histológicas son la fibrosis de túbulos seminíferos, alteración de células de Sertoli y Leydig, engrosamiento de la membrana basal, edema intersticial y aumento de mastocitos en el parénquima testicular ${ }^{(8-10)}$.

La localización de supraescrotal del teste puede hallarse en la criptorquidia congénita, pero también en la criptorquidia adquirida y en el teste en ascensor o teste retráctil. La criptorquidia adquirida tiene una prevalencia estimada por Hack del $2,2 \%$ en la población de 9 años y del 1,1\% en la población de 13 años. En un estudio de Guven se encontró que el 45\% de las orquidopexias tardías ocurren en pacientes con antecedente de descenso testicular normal, y van der Plas encontró que hasta un 66\% de las orquidopexias tardías tenían antecedente de descenso normal en historias clínicas ${ }^{(11-13)}$. Virtanen, en su revisión sobre criptorquidia y fertilidad, defiende que las alteraciones histológicas de la criptorquidia adquirida y congénita son iguales ${ }^{(14)}$. Está claro que la localización supraescrotal se acompaña de una alteración histológica, que se manifiesta clínicamente con una disminución del volumen testicular y alteración del espermiograma. Pretendemos estudiar el impacto a largo plazo que la criptorquidia tiene sobre el volumen testicular final y las perspectivas que puede tener un teste de compensar su crecimiento, así como también si estas alteraciones de volumen se correlacionan con una alteración en la paternidad. Nuestra hipótesis es que los testes en posición anómala presentan una reducción y afectación permanente de su volumen y funcionamiento.

\section{MATERIAL Y MÉTODOS}

\section{Ámbito y sujetos de estudio}

Pacientes nacidos entre los años 1961 y 1985, intervenidos de criptorquidia en el Servicio de Cirugía Pediátrica del Hospital Universitario Miguel Servet y que fueron objeto de un estudio de fertilidad en el año 1999. De estos pacientes, disponemos de datos clínicos y quirúrgicos, analíticos y anatomopatológicos desde la niñez. También disponemos de ecografías testiculares postoperatorias de control. Se calculó el tamaño testicular mediante mediciones ecográficas y la fórmula de elipsoide en la que la constante 0,52 se multiplica con el resultado de la longitud multiplicado por la anchura al cuadrado (0,52 x [ $\left.\left.\mathrm{L} \mathrm{x} \mathrm{A}^{2}\right]\right)$, y los volúmenes se describen en centímetros cúbicos.

Se envió una encuesta por correo a 258 pacientes intervenidos en nuestro servicio de criptorquidia y que forman parte de la base de datos antes comentada. Recibimos respuesta de 97 pacientes. A los 161 pacientes restantes realizamos una llamada telefónica. Un total de 60 pacientes accedieron a realizar una encuesta telefónica. En total captamos 157 casos $(60,85 \%)$.

\section{Diseño y metodología estadística}

Se realizó un estudio de cohortes retrospectivo que compara, mediante una encuesta, la paternidad de un grupo con el factor de exposición común de criptorquidia, con otro grupo equivalente de individuos sin dicha afectación. La segunda parte es el análisis estadístico de las ecografías testiculares realizadas a los individuos adultos intervenidos de criptorquidia en relación con los datos de la encuesta administrada a los mismos.

En el análisis descriptivo de los datos, las variables cualitativas se presentan mediante la distribución de frecuencias de los porcentajes de cada categoría. Las variables estudiadas cuantitativas son exploradas con la prueba de conformidad de Kolmogorov-Smirnov (prueba de bondad de ajuste a una distribución normal).

\section{Análisis bivariante. Comparación entre variables}

La asociación entre los factores se investiga mediante pruebas de contraste de hipótesis. Los efectos se consideran significativos si $\mathrm{p}<0,05$, y los valores de $\mathrm{p}$ son de dos colas.

Se utilizó para realizar el análisis estadístico el programa SPSS v.18.0.0, se contó con el apoyo estadístico del SAME (Servicio de Apoyo Metodológico y Estadístico) del Instituto Aragonés de Ciencias de la Salud.

\section{Consideraciones éticas}

El estudio cuenta con el consentimiento informado de todos los participantes y ha sido aprobado por el CEICA (Comité de Ética en la Investigación de la Comunidad Autónoma de Aragón). $\mathrm{N}^{\circ} \mathrm{CP} 12 / 2014$.

Los datos forman parte de la tesis doctoral presentada en 2017 por Rafael Fernández Atuan, en Zaragoza, con título: "Estudio de la fertilidad en pacientes operados de criptorquidia".

\section{RESULTADOS}

Las edades de los encuestados están entre 31 y 55 años, con una media de edad de 41,9 años. Las ecografías testiculares se realizaron a un total de 216 unidades testiculares entre 1 y 20 años después de la orquidopexia, con un promedio de 14,9 años. 


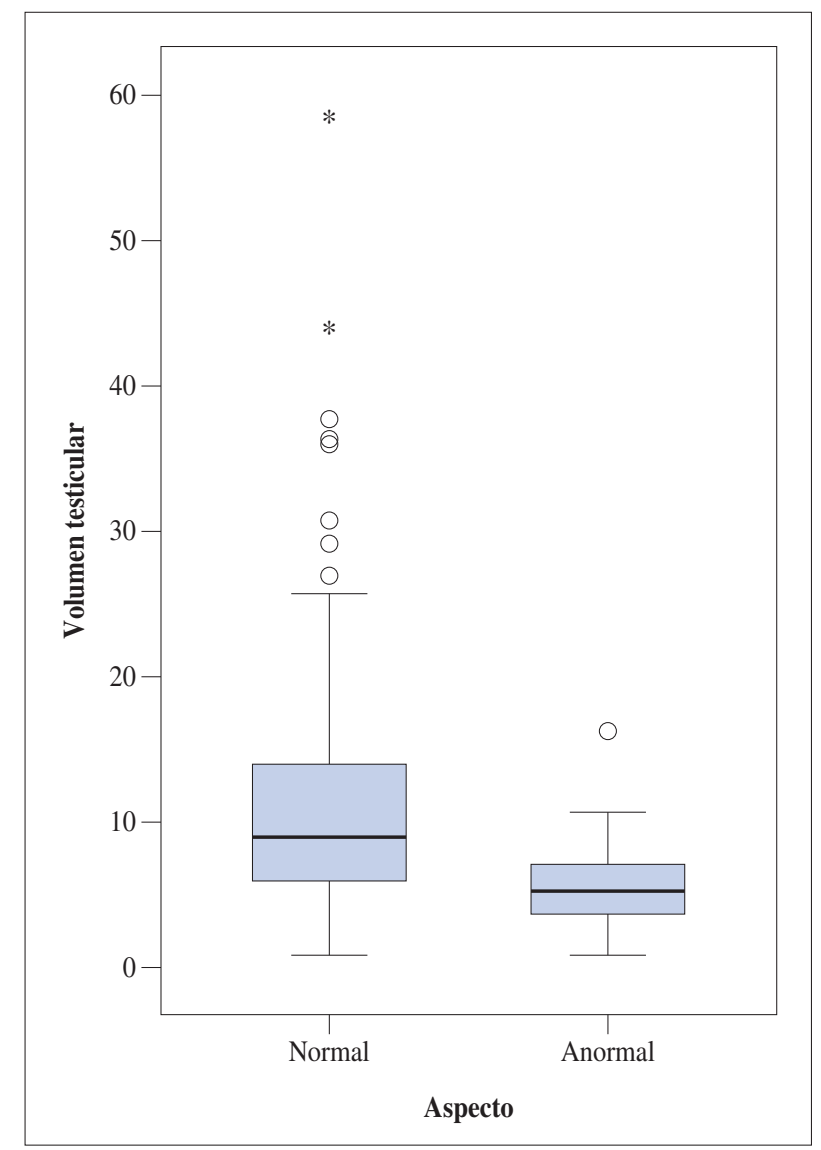

Figura 1. Volumen testicular con relación al aspecto de la unión epidídimo testicular. Se observan diferencias estadísticamente significativas en la distribución del volumen testicular según el aspecto del teste (volumen testicular en centímetros cúbicos).

Un total de $120(76,43 \%)$ son pacientes con afectación unilateral y $37(23,57 \%)$ son bilaterales. El 14,4\% de los testes intervenidos tienen un aspecto anormal en la intervención quirúrgica. Definiendo anormal como disyunción epidídimotesticular completa. Se observa una diferencia estadísticamente significativa $(\mathrm{p}<0,038)$ en la distribución del volumen testicular según el aspecto del teste (Fig. 1).

La edad media de tratamiento quirúrgico es de 6,2 años. $\mathrm{Al}$ analizar la edad de tratamiento quirúrgico con el volumen testicular se observa correlación lineal entre la edad de tratamiento quirúrgico y un menor volumen testicular en la población total. Esta correlación no alcanza significancia estadística en nuestro estudio (Fig. 2, Tabla I).

$\mathrm{Al}$ analizar el volumen testicular entre los testes intervenidos y los contralaterales no operados, se observan diferencias estadísticamente significativas en la distribución del volumen testicular según grupos de operados (testes retenidos) y no operados (contralaterales) (Fig. 3).

Al comparar el volumen de los testes de pacientes con criptorquidia unilateral con los de pacientes con afectación bilateral se observan diferencias estadísticamente significa-

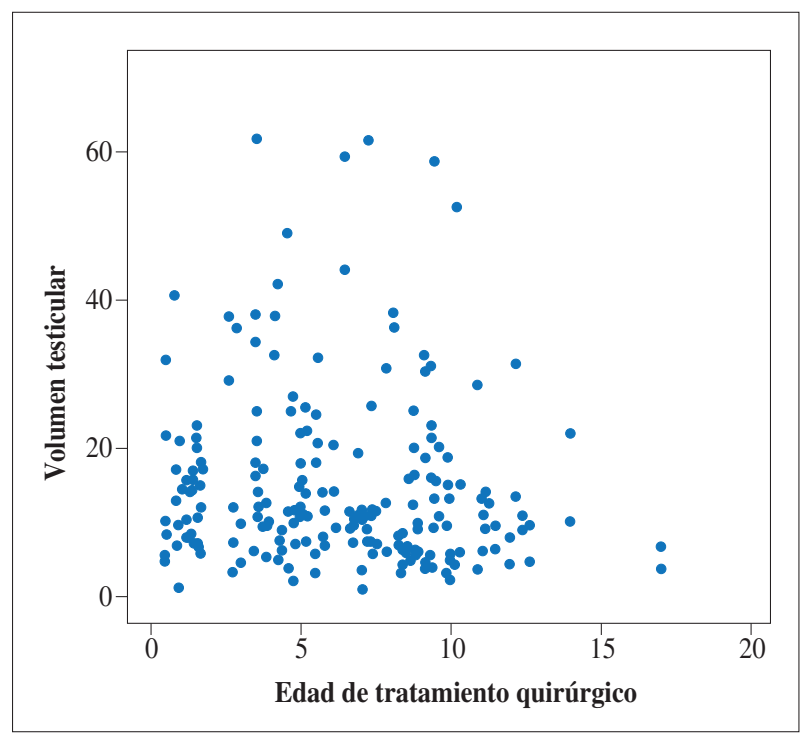

Figura 2. Análisis lineal entre edad de tratamiento quirúrgico y volumen testicular.

Tabla I. Análisis de edad de tratamiento quirúrgico y volumen testicular.

\begin{tabular}{|c|c|c|c|c|}
\hline & & \multicolumn{3}{|c|}{ Volumen testicular } \\
\hline & & $\begin{array}{c}\text { Población } \\
\text { total }\end{array}$ & Contralateral & Operado \\
\hline \multirow{2}{*}{$\begin{array}{l}\text { Edad } \\
\text { tratamiento } \\
\text { quirúrgico }\end{array}$} & $\begin{array}{c}\text { Correlación } \\
\text { Pearson }\end{array}$ & $-0,11$ & $-0,14$ & $-0,02$ \\
\hline & p-valor & $>0,05$ & $>0,05$ & $>0,05$ \\
\hline \multicolumn{5}{|c|}{$\begin{array}{l}\text { Se observa una correlación negativa de Pearson, aunque esta correlación lineal } \\
\text { entre la edad de tratamiento quirúrgico y el volumen testicular en la población } \\
\text { total no es estadísticamente significativa en nuestro estudio (volumen testicular } \\
\text { en centímetros cúbicos y edad en años). }\end{array}$} \\
\hline
\end{tabular}

tivas en la distribución del volumen testicular según grupos unilaterales y bilaterales. Los testes en pacientes con afectación unilateral tienen mayor volumen (Fig. 4).

No se observa relación estadística entre el volumen testicular y los índices de paternidad de los pacientes operados (Tabla II). El tiempo para lograr el embarazo en nuestro estudio es de 10,6 meses para los pacientes con afectación unilateral, 15,8 meses para el grupo bilateral y 8,1 meses para el grupo control.

Del número total de pacientes, solo 19 pacientes respondieron que requirieron tratamiento de fertilidad de algún tipo. Al consultar sobre el tipo de tratamiento, solo respondieron 18 personas: el $44,4 \%$ de los pacientes que precisaron tratamiento para la fertilidad utilizaron el método FIV para quedar embarazados, 27,8\% hormonas masculinas, $11,1 \%$ ICSI (inyección intracitoplasmática de semen en ingles) y $16,7 \%$ el tratamiento fue necesario para la pareja femenina solamente. 


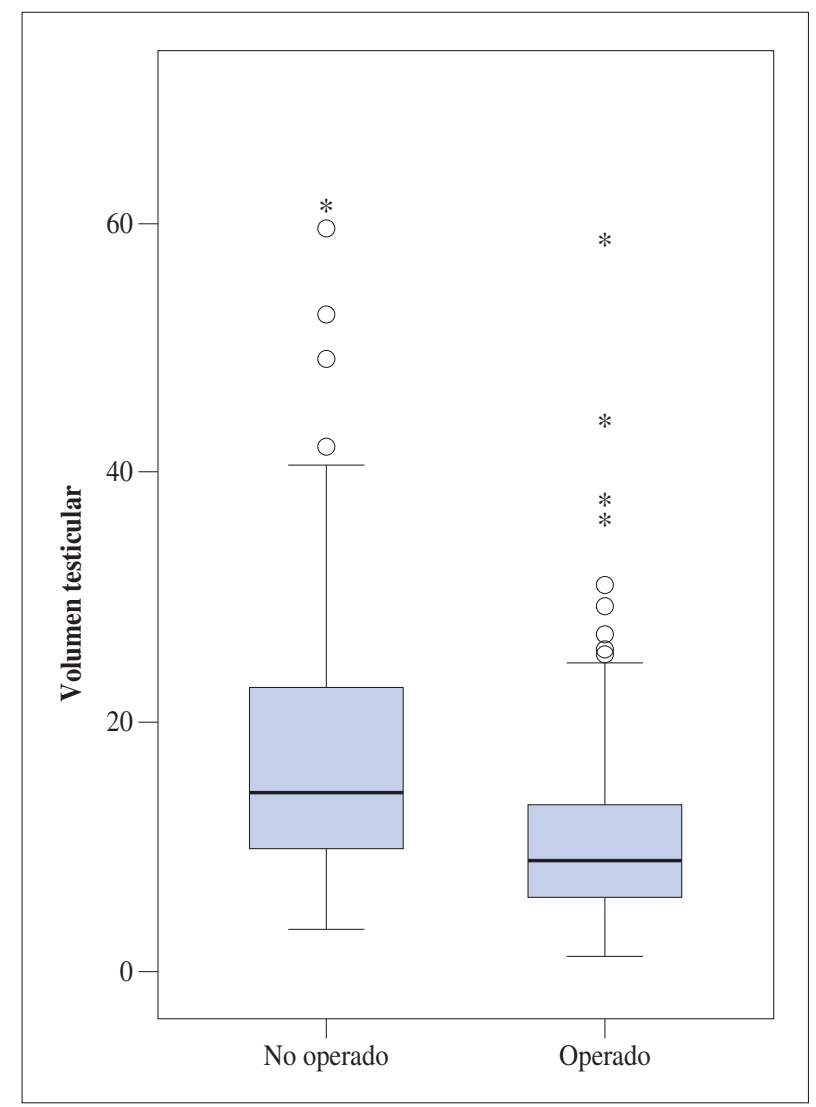

Figura 3. Relación entre volumen testicular de testes operados y no operados. Se observa diferencias estadísticamente significativas en la distribución del volumen testicular según grupos de operados (testes retenidos) y no operados (contralaterales) (volumen testicular en centímetros cúbicos).

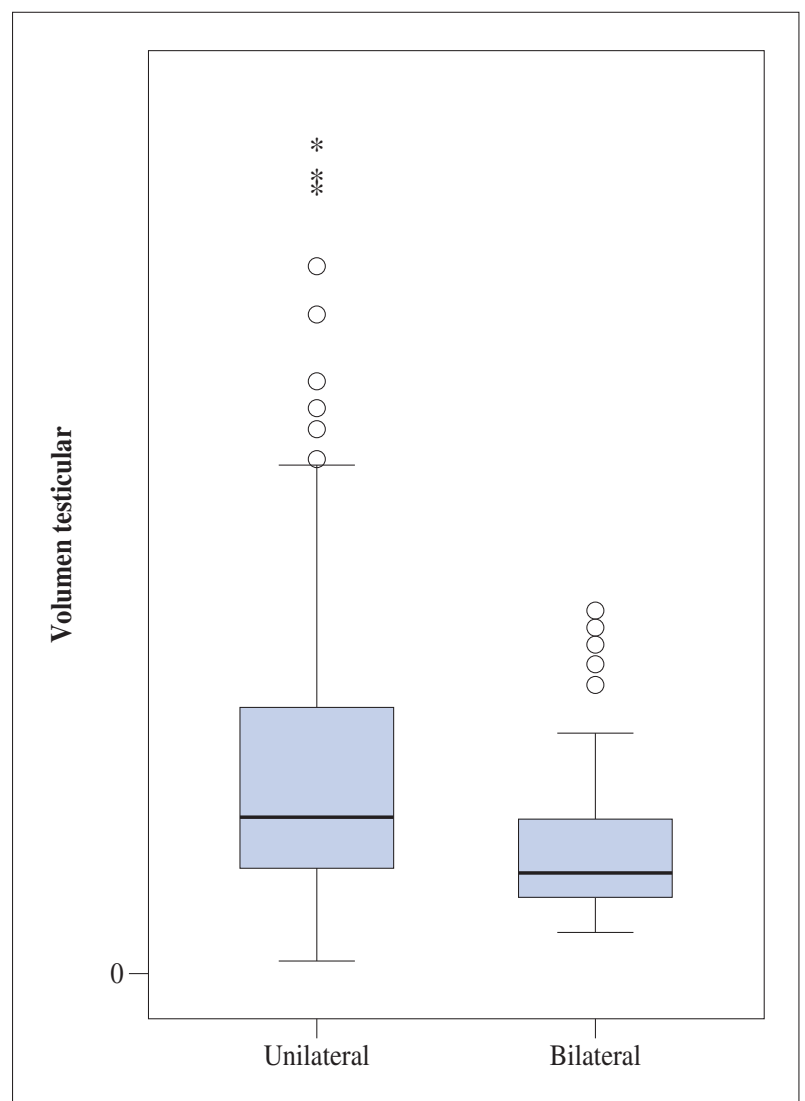

Figura 4. Relación entre el volumen testicular y afectación unilateral o bilateral. Los testes en pacientes con afectación unilateral tienen mayor volumen testicular (volumen testicular en centímetros cúbicos).

Tabla II. Análisis del volumen testicular y paternidad.

\begin{tabular}{|c|c|c|c|c|c|}
\hline & & Población total & No tiene hijos & Sí tiene hijos & p-valor \\
\hline \multirow{2}{*}{ Volumen testículos operados } & Media & 12,07 & 12,10 & 12,25 & \multirow{2}{*}{$>0,05$} \\
\hline & Desviación típica & 10,45 & 11,60 & 9,30 & \\
\hline \multirow{2}{*}{ Volumen testículos no operados } & Media & 18,78 & 18,51 & 19,34 & \multirow{2}{*}{$>0,05$} \\
\hline & Desviación típica & 12,91 & 11,70 & 14,36 & \\
\hline
\end{tabular}

\section{DISCUSIÓN}

En nuestro estudio, el teste se identificaba como normal o anormal según sus características clínicas en el momento de la cirugía. Estas características no abarcan solo el aspecto macroscópico del parénquima testicular, sino también y de manera muy importante el aspecto del epidídimo en relación con el testículo. Normalmente, el epidídimo debe estar completamente unido al parénquima testicular. Sin embargo, para este estudio, y por efecto de la variabilidad en la apreciación entre los cirujanos, solo se consideraron anormales los testes con una disyunción epidídimo testicular completa, que en nuestro estudio es de $14,4 \%$. Encontramos una diferencia estadísticamente significativa entre el teste anormal con dicha disociación y el volumen testicular $(p=0,038)$. En un estudio de Kim, que evalúa el aspecto testicular comparando pacientes con criptorquidia e hidrocele, encuentra una disyunción completa de un $12,3 \%{ }^{(15)}$. Este estudio encuentra que todos los testes intraabdominales presentan alteración del epidídimo y que a mayor altura testicular, más alteración en la unión epidídimo-testicular ${ }^{(15)}$. No encontramos artículos que compararan estado del epidídimo con volumen testicular. 
La edad de intervención quirúrgica, el volumen testicular y su relación con la fertilidad han sido desde hace muchos años objeto de investigación. En nuestro estudio se observa una correlación negativa entre el volumen testicular y la edad de tratamiento quirúrgico sin llegar a significancia estadística. Es importante resaltar el control testicular ecográfico de 14,9 años después de la intervención, ya que el teste intervenido puede compensar y crecer, nuestra edad media de intervención es alta, con 6,2 años, siendo la edad de intervención recomendada actualmente entre 12 y 30 meses. Este resultado es igual al que obtuvo Taskinen en su estudio, con un volumen testicular menor en los pacientes intervenidos con cuatro años en comparación con los intervenidos a mayor edad, sin alcanzar significación estadística ${ }^{(16)}$. Otros investigadores sí encuentran diferencias significativas entre el volumen testicular de los testes no descendidos intervenidos a los nueve meses en comparación con los intervenidos a los tres años, con un seguimiento de estos pacientes hasta los cinco años en ambos grupos y, por tanto, el tiempo de seguimiento que permitiera la recuperación del teste operado fue diferente en ambos grupos ${ }^{(17)}$. Durell encontró, con un tiempo de seguimiento de 6,9 meses, que hasta un 20\% de los testículos compensan su crecimiento hasta ser comparables con los contralaterales cuando se realiza la orquidopexia alrededor de los 12 meses, siendo valorados los testículos subjetivamente por los investigadores ${ }^{(18)}$. La atrofia testicular se reporta de forma significativa en los pacientes operados después de los 13 meses en comparación con los operados antes de esta edad ${ }^{(19)}$. Cuando Carson realizó un análisis de regresión comparando la edad de tratamiento quirúrgico y la atrofia testicular postoperatoria, corrigiendo por localización testicular, no encontró diferencias significativas, sugiriendo que el impacto de la localización extraabdominal es menor que la edad de intervención ${ }^{(16,19)}$. Es importante resaltar que actualmente la edad en nuestro servicio de intervención quirúrgica se sitúa entre los 13 y 30 meses de edad.

No encontramos correlación significativa entre el volumen testicular y la paternidad. Lee, en su estudio con 166 pacientes con orquidopexia unilateral, no encontró diferencias en paternidad en relación con volumen testicular, su estudio se basaba en la apreciación clínica del tamaño testicular por el equipo quirúrgico ${ }^{(20)}$. El volumen testicular no parece correlacionar con el número de células germinales y por tanto no puede usarse con un parámetro fiable para predecir capacidad reproductiva, aunque el volumen se asocia con un claro deterioro de la calidad del semen ${ }^{(21)}$. Ates, en su estudio, encontró que la única variable que se relacionaba a la calidad del semen en pacientes adultos era el volumen testicular del teste descendido ${ }^{(20-22)}$. En el caso de la criptorquidia bilateral no encontramos diferencias estadísticamente significativas respecto a la paternidad entre los pacientes con criptorquidia bilateral y el grupo control. Se reporta, en la literatura resultados similares y atribuyeron este resultado al bajo número de pacientes con criptorquidia bilateral incluidos en el estudio ${ }^{(23)}$. En nuestro caso, son 37 pacientes con afectación bilateral y en el caso del estudio de van Brakel participaron siete pacien- tes ${ }^{(23)}$. Estudios con más pacientes con criptorquidia bilateral sí reportan una reducción importante y significativa de los parámetros de paternidad. Aproximadamente un 65,3\% de los pacientes bilaterales con intentos de paternidad tuvieron éxito, lo que es, de manera estadísticamente significativa, un porcentaje menor que en pacientes con criptorquidia unilateral o controles ${ }^{(24)}$. En los pacientes bilaterales se reporta que a quienes se les realizó orquidopexia precoz (entre 10 meses y 3 años) el número de espermatozoides era normal en el 76\%, en comparación con pacientes intervenidos posteriormente con un número de espermatozoides normal en solo el 26\%(24,25). Una revisión reciente del manejo de la criptorquidia reporta que los pacientes afectos de forma bilateral presentan en un $75 \%$ oligospermia y un $42 \%$ azoospermia $^{(26)}$.

\section{CONFLICTOS DE INTERÉS Y LIMITACIONES DEL ESTUDIO}

Los autores del presente estudio no tenemos conflictos de interés. Las limitaciones de este estudio son su naturaleza retrospectiva y la inclusión de pacientes con criptorquidia congénita con criptorquidia adquirida por la naturaleza de recopilación de datos.

\section{CONCLUSIONES}

Los testes intervenidos inevitablemente sufren un impacto en su crecimiento final en comparación con el crecimiento del teste contralateral descendido y en relación con los testes de la población normal. Este impacto se correlaciona con la edad de tratamiento quirúrgico en nuestro estudio y otros, aunque en nuestro caso no logramos significación estadística.

Los pacientes con afectación bilateral y disyunción epidídimo testicular tienen peor capacidad de compensación de crecimiento y, por tanto, un menor volumen testicular. Los pacientes con afectación bilateral precisaron de más tiempo para alcanzar el embarazo.

No encontramos asociación entre el volumen testicular y la paternidad, por lo que el volumen testicular no parece ser un método fiable para determinar la fertilidad. Los pacientes con afectación bilateral precisaron de más tiempo para alcanzar el embarazo.

Más estudios de seguimiento a largo plazo son necesarios para corroborar los resultados.

\section{BIBLIOGRAFÍA}

1. Hutson JM, Balic A, Nation T, Southwell B. Cryptorchidism. Semin Pediatr Surg. 2010; 19(3): 215-24.

2. Virtanen HE, Bjerknes R, Cortes D, Jørgensen N, Rajpert-De Meyts E, Thorsson AV, et al. Cryptorchidism: classification, prevalence and long-term consequences. Acta Paediatr. 2007; 96(5): 611-6. 
3. Barthold JS, González R. The epidemiology of congenital cryptorchidism, testicular ascent and orchiopexy. J Urol. 2003; 170(6 Pt 1): $2396-401$.

4. Holland AJ, Nassar N, Schneuer FJ. Undescended testes: an update. Curr Opin Pediatr. 2016; 28(3): 388-94.

5. Schneuer FJ, Holland AJ, Pereira G, Jamieson S, Bower C, Nassar N. Age at Surgery and Outcomes of an Undescended Testis. Pediatrics. 2016; 137(2): e20152768.

6. Hadziselimovic F, Hocht B, Herzog B, Buser MW. Infertility in cryptorchidism is linked to the stage of germ cell development at orchidopexy. Horm Res. 2007; 68(1): 46-52.

7. Verkauskas G, Malcius D, Eidukaite A, Vilimas J, Dasevicius D, Bilius V, et al. Prospective study of histological and endocrine parameters of gonadal function in boys with cryptorchidism. J Pediatr Urol. 2016; 12(4): 238.e1-6.

8. Acikgoz A, Asci R, Aydin O, Çavus H, Donmez G, Buyukalpelli R. The role of ketotifen in the prevention of testicular damage in rats with experimental unilateral undescended testes. Drug Des Devel Ther. 2014; 8: 2089-97.

9. Li R, Thorup J, Sun C, Cortes D, Southwell B, Hutson J. Immunofluorescent analysis of testicular biopsies with germ cell and Sertoli cell markers shows significant MVH negative germ cell depletion with older age at orchiopexy. J Urol. 2014; 191(2): 458-64.

10. Koni A, Ozseker HS, Arpali E, Kilinc E, Dogan HS, Akyol A, et al. Histopathological evaluation of orchiectomy specimens in 51 late postpubertal men with unilateral cryptorchidism. J Urol. 2014; 192(4): 1183-8.

11. Hack WW, Sijstermans K, van Dijk J, van der Voort-Doedens LM, de Kok ME, Hobbelt-Stoker MJ. Prevalence of acquired undescended testis in 6-year, 9-year and 13-year-old Dutch schoolboys. Arch Dis Child. 2007; 92(1): 17-20.

12. Guven A, Kogan BA. Undescended testis in older boys: further evidence that ascending testes are common. J Pediatr Surg. 2008; 43(9): 1700-4.

13. van der Plas EM, van Brakel J, Meij-de Vries A, de Muinck KeizerSchrama SM, Hazebroek FW, Hack WW, et al. Acquired undescended testes and fertility potential: is orchiopexy at diagnosis better than awaiting spontaneous descent? Andrology. 2015; 3(4): 677-84.

14. Virtanen HE, Toppari J. Cryptorchidism and Fertility. Endocrinol Metab Clin North Am. 2015; 44(4): 751-60.
15. Kim SO, Na SW, Yu HS, Kwon D. Epididymal anomalies in boys with undescended testis or hydrocele: Significance of testicular location. BMC Urol. 2015; 15: 108.

16. Taskinen S, Wikström S. Effect of age at operation, location of testis and preoperative hormonal treatment on testicular growth after cryptorchidism. J Urol. 1997; 158(2): 471-3.

17. Kollin C, Granholm T, Nordenskjöld A, Ritzén EM. Growth of spontaneously descended and surgically treated testes during early childhood. Pediatrics. 2013; 131(4): e1174-80.

18. Durell J, Johal N, Burge D, Wheeler R, Griffiths M, Kitteringham L, et al. Testicular atrophy following paediatric primary orchidopexy: A prospective study. J Pediatr Urol. 2016; 12(4): 243.e1-4.

19. Carson JS, Cusick R, Mercer A, Ashley A, Abdessalam S, Raynor $\mathrm{S}$, et al. Undescended testes: does age at orchiopexy affect survival of the testis? J Pediatr Surg. 2014; 49(5): 770-3.

20. Lee PA, Coughlin MT, Bellinger MF. No relationship of testicular size at orchiopexy with fertility in men who previously had unilateral cryptorchidism. J Urol. 2001; 166(1): 236-9.

21. Noh PH, Cooper CS, Snyder HM, Zderic SA, Canning DA, Huff DS. Testicular volume does not predict germ cell count in patients with cryptorchidism. J Urol. 2000; 163(2): 593-6.

22. Ates F, Soydan H, Okçelik S, Çırakoglu A, Yılmaz I, Malkoç E, et al. Clinical and histopathological results of the adult patients with unilateral cryptorchidism. Turk J Urol. 2016; 42(2): 74-9.

23. van Brakel J, Kranse R, de Muinck Keizer-Schrama SM, Hendriks $\mathrm{AE}$, de Jong FH, Bangma $\mathrm{CH}$, et al. Fertility potential in men with a history of congenital undescended testes: a long-term follow-up study. Andrology. 2013; 1(1): 100-8.

24. Lee PA, Coughlin MT. Fertility after bilateral cryptorchidism. Evaluation by paternity, hormone, and semen data. Horm Res. 2001; 55(1): 28-32.

25. Thorup J, McLachlan R, Cortes D, Nation TR, Balic A, Southwell $\mathrm{BR}$, et al. What is new in cryptorchidism and hypospadias--a critical review on the testicular dysgenesis hypothesis. J Pediatr Surg. 2010; 45(10): 2074-86.

26. Radmayr C, Dogan HS, Hoebeke P, Kocvara R, Nijman R, Stein $\mathrm{R}$, et al. Management of undescended testes: European Association of Urology/European Society for Paediatric Urology Guidelines. J Pediatr Urol. 2016; 12(6): 335-43. 